\title{
Analysing a mechanism of failure in retrieved magnetically controlled spinal rods
}

\author{
Vasiliki C. Panagiotopoulou ${ }^{1,2} \cdot$ Stewart K. Tucker $^{3} \cdot$ Robert K. Whittaker $^{1}$ • \\ Harry S. Hothi ${ }^{1} \cdot$ Johann Henckel $^{1,2} \cdot$ Julian J. H. Leong ${ }^{1,2} \cdot$ Thomas Ember $^{3,4}$. \\ John A. Skinner ${ }^{1,2}$ Alister J. Hart ${ }^{1,2}$
}

Received: 1 July 2016/Revised: 19 December 2016/Accepted: 25 December 2016/Published online: 19 January 2017 (c) The Author(s) 2017. This article is published with open access at Springerlink.com

\begin{abstract}
Purpose We aim to describe a mechanism of failure in magnetically controlled growth rods which are used for the correction of the early onset scoliosis.

Methods This retrieval study involved nine magnetically controlled growth rods, of a single design, revised from five patients for metal staining, progression of scoliosis, swelling, fractured actuator pin, and final fusion. All the retrieved rods were radiographed and assessed macroscopically and microscopically for material loss. Two implants were further analysed using micro-CT scanning and then sectioned to allow examination of the internal mechanism. No funding was obtained to analyse these implants. There were no potential conflicts interests.

Results Plain radiographs revealed that three out of nine retrieved rods had a fractured pin. All had evidence of surface degradation on the extendable telescopic rod. There was considerable corrosion along the internal mechanism. Conclusions We found that a third of the retrieved magnetically controlled growth rods had failed due to pin fracture secondary to corrosion of the internal mechanism. We recommend that surgeons consider that any inability of magnetically controlled growth rods to distract may be due to corrosive debris building up inside the mechanism, thereby preventing normal function.
\end{abstract}

Vasiliki C. Panagiotopoulou

v.panagiotopoulou@ucl.ac.uk

1 Institute of Orthopaedics and Musculoskeletal Science, Royal National Orthopaedic Hospital, University College London, Brockley Hill, Stanmore, Middlesex HA7 4LP, UK

2 The Royal National Orthopaedic Hospital, Stanmore, UK

3 Great Ormond Street Hospital for Children, London, UK

4 Guy's and St Thomas NHS Foundation Trust, London, UK
Keywords Early onset scoliosis - Magnetically controlled growth rods $\cdot$ Spine retrievals $\cdot$ Spine implants $\cdot$ Corrosion

\section{Introduction}

Lateral spine deformation in paediatric patients aged less than 10 years is diagnosed as the early onset scoliosis (EOS) [1]. If the condition is left untreated, this spine deformation worsens, affecting not only development and growth, but also lung function [2]. The latter result in cardiorespiratory problems and often mortality [3]. Treatment can either be surgical (use of spine implants) or nonsurgical (casting and bracing), depending on the age of the patient and the severity of EOS $[4,5]$.

Spinal rods used for the surgical treatment of the EOS are divided into two categories: (1) traditional growing systems (that require a small incision to manually lengthen the rod) and (2) magnetically controlled growth rods (MCGRs) that are lengthened through the use of an external magnet. MCGRs are gaining in popularity among patients and surgeons as they are the least invasive technique for patients with a high degree scoliosis [6-11], both for primary operations and conversion cases from the traditional growing rods [12]. Once implanted (see Fig. 1), MCGRs are elongated non-invasively every 3 months using an external remote controller (the external magnet) in an outpatients' clinic, while the patient is conscious. The lengthening procedure lasts approximately $40 \mathrm{~s}$ per rod and the total length is measured using a plain radiograph [8]. Recent studies have also investigated the reliability of other imaging modalities, including ultrasound [13-15].

Recently, one paper with two case reports was published suggesting that the internal mechanism of a certain design 


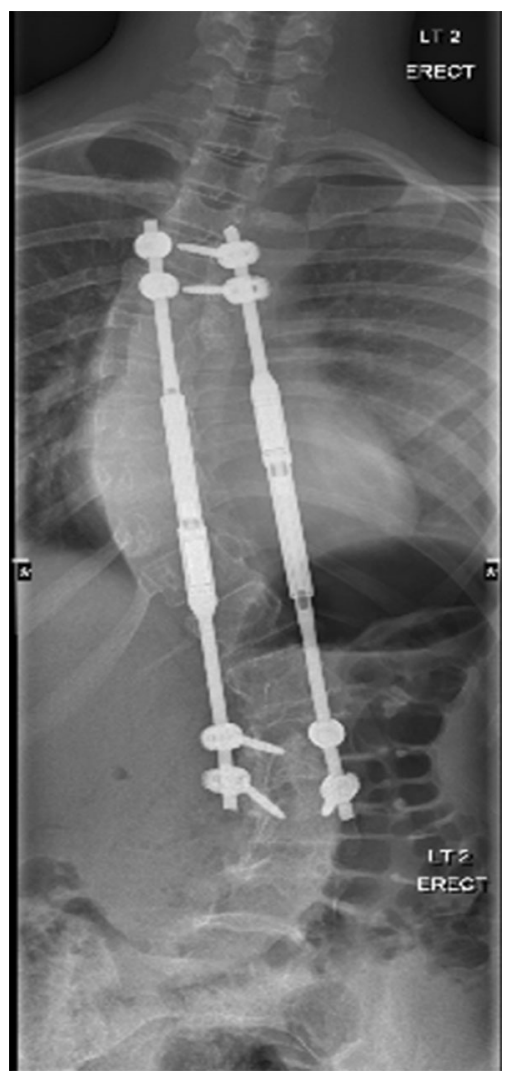

Fig. 1 MCGR in situ

of spinal rod (MAGnetic Expansion Control, Nuvasive Inc.) can fracture, resulting in the failure of the lengthening mechanism [16]. This is further supported by a 4 year follow-up study that included one case of a fractured pin within the rod [17]. This mechanical distraction failure was also noted in a special article for the device [18]. Comparison between primary and conversion cases showed no significant difference regarding the complications, where in five primary patients and 1 conversion patient $(p=0.20)$, the rods failed to distract [12]. No official data have been published for revision rates of this design; however, it is estimated that unplanned revision reaches $22 \%$ of the cases, based on figures presented at Annual Meeting of the British Scoliosis Society [19].

The aim of our study was to (1) determine the prevalence of MCGRs that had failed due to a fractured pin in our retrieval collection and (2) understand the mechanism of failure.

\section{Materials and methods}

This was a retrospective study involving nine retrieved MCGRs, received between 2015 and 2016 from five patients, three hospitals, and three surgeons. All implants were the same design (MAGnetic Expansion Control, Nuvasive Inc.).

Implants were retrieved from 4 females and 1 male with a median (range) age of 10 (9-12) years with a median (range) time of implantation of 25.5 months (18-32), Table 1. Six of the MCGRs were deemed thee arly revisions due to patient reactions to the implants (black staining of the skin on the back, swelling, and progression of scoliosis). 1 was removed for a fractured actuator pin visible on pre-revision radiographs and 2 were removed to accommodate final fusion.

\section{Plain radiographs}

We hypothesized that the failure of the MCGRs was caused by fracture of the pin in the actuator. Our primary outcome measure was evidence of pin fracture on plain radiographs taken of the explanted device [16], Fig. 2b, c.

\section{Macroscopic inspection}

All implants were visually assessed for macroscopic signs of material loss, using an adaptation of a published grading score (from 0 to 3 , see Table 2) [19]. The score was used for classifying the signs of corrosion for spine rods at junctions with hooks and connectors. Rods were divided into five regions of interest which were individually assessed for surface damage and scored from 0 (no sign of damage) to 3 (severe metal loss). Junctions, bent regions, and the full length of the actuator were selected as sites of interest, Fig. 3.

\section{Microscopic inspection}

Microscopic analysis of the rods was carried out using a Keyence VHX-700F series (Keyence Co., Japan) digital microscope, with a magnification range of $20 \times$ to $1000 \times$.

\section{Detailed forensic analysis of the internal mechanism}

We selected one rod that had clear evidence of pin fracture and one in which the pin was intact. We performed detailed forensic testing, including Micro-CT (mCT), mechanical sectioning, optical microscopy, scanning electron microscopy (SEM), and Energy Dispersive X-ray Spectrometry (EDX).

\section{Micro-CT}

mCT was performed on the two selected rods using a Nikon Metrology 225 Micro-CT Scanner. Each rod had two separate scans, one at the proximal and one at the distal 
Table 1 Demographic data for patients and implants included in the study

\begin{tabular}{llclll}
\hline Patient & Number of rods used & Age at primary (years) & Gender & Implantation time (months) & Reason for revision \\
\hline 1 & 2 & 9 & Female & 24 & Metal staining of the skin \\
2 & 2 & 10 & Female & 27 & Progression of Scoliosis \\
3 & 2 & 12 & Female & 18 & Swelling \\
4 & 1 & 10 & Female & 32 & Broken pin \\
5 & 2 & 10 & Male & 31 & Final fusion \\
\hline
\end{tabular}

a

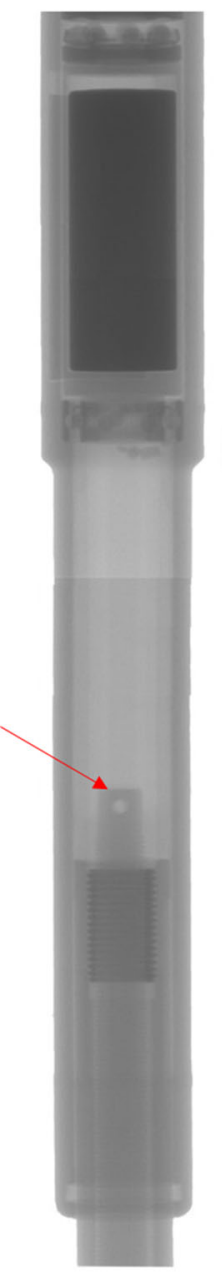

$1 \mathrm{~A}$

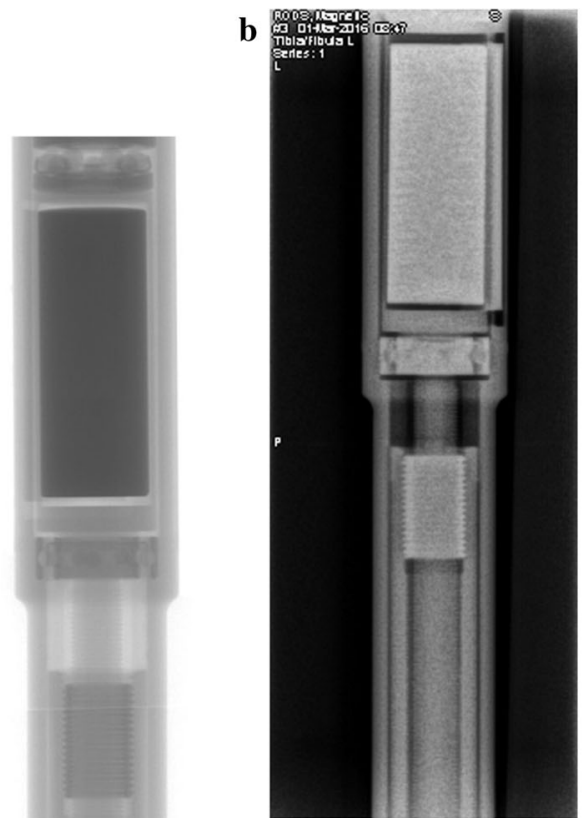

$2 \mathrm{~A}$

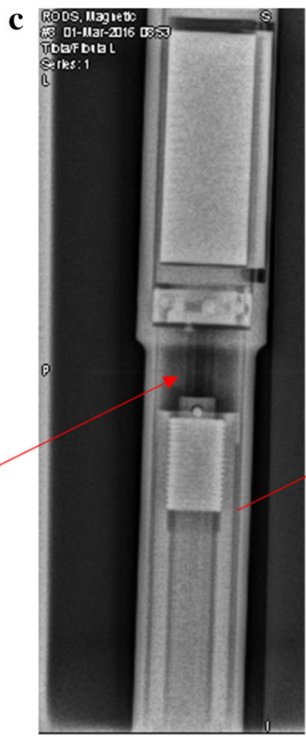

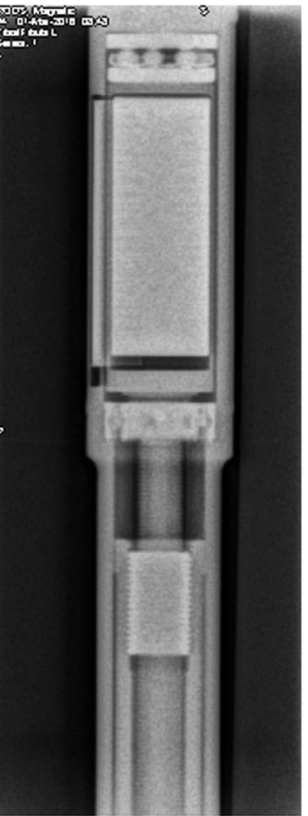

2B

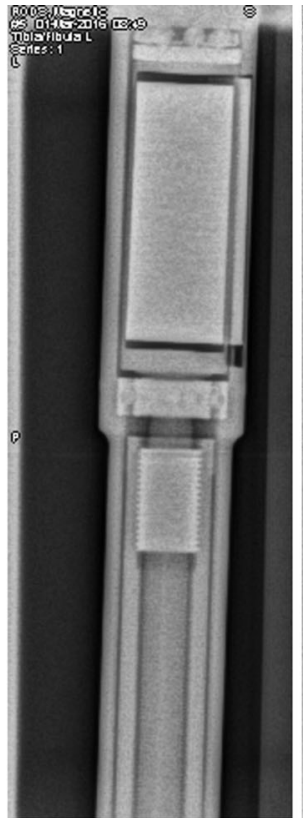

$3 \mathrm{~A}$

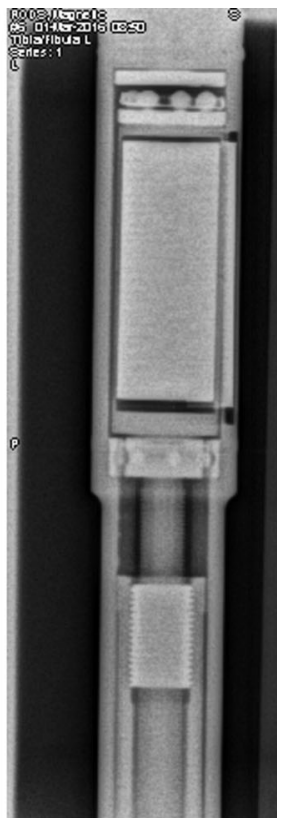

$3 \mathrm{~B}$

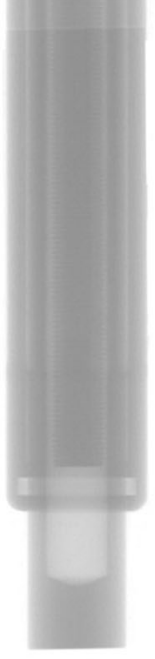

$1 \mathrm{~B}$

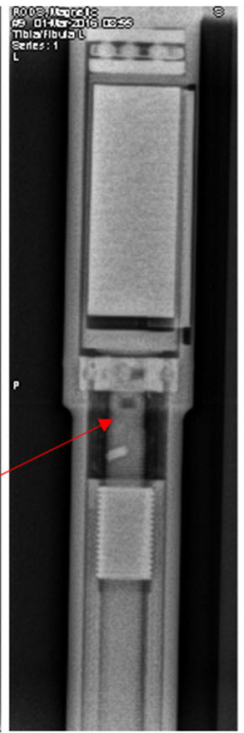

$5 \mathrm{~A}$

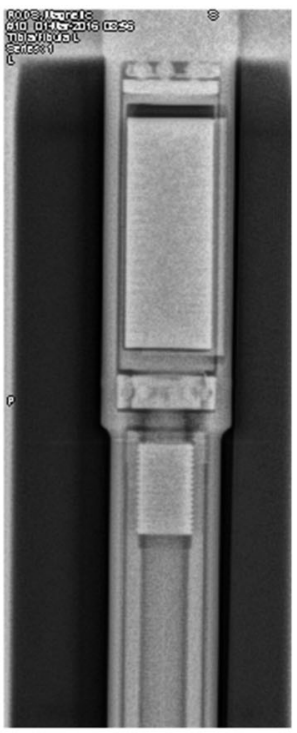

$5 \mathrm{~B}$

Fig. 2 a High power radiographs of two retrieved MCGRs. In rod $1 \mathrm{~A}$, the internal mechanism is no longer in place (indicated by the red arrow), while rod 1B appears intact. b Plain radiographs of four

retrieved rods, all of them appear intact. $\mathbf{c}$ Plain radiographs of three retrieved rods, two of them having a fractured mechanism as indicated by the red arrows, while the rest remains intact 
Table 2 Criteria used to grade surface material loss on the spinal rods

\begin{tabular}{ll}
\hline Grade & Criteria \\
\hline 0 & No sign of corrosion \\
1 & Surface discoloration \\
2 & Superficial metal loss \\
3 & Severe metal loss \\
\hline Akazawa et al. [19]
\end{tabular}

end of the MCGR. Each scan contained 835 frames that were reconstructed using the Nikon Metrology software.

\section{Mechanical sectioning}

The MCGRs were secured by a collet and sectioned using an undercut tool made of tungsten carbide with a width of $1.2 \mathrm{~mm}$. mCT imaging was used to identify the areas of interest within the MCGR, as well as the best sites to section the implant to ensure that the internal mechanism was not damaged during the sectioning.

\section{SEM/EDX}

A scanning electron microscope (Hitachi S-3400 N) was used for a further detailed microscopic analysis of the retrieved components using $15 \mathrm{kV}$ at a working distance of $10 \mathrm{~mm}$. Energy Dispersive X-ray Spectrometry (Oxford Instruments) was used in combination with the SEM to identify the elemental composition.

\section{Results}

\section{All implants}

\section{Plain radiograph}

Plain radiographs showed evidence of dislocation of the thread mechanism of the telescopic components in three cases.
Fig. 3 Image of an explanted MCGR, with labelling of the main parts of the rod (actuator and extendable telescopic rod) and with referencing of the five areas of interest used for comparison of surface damage findings for each rod

Fig. 4 Images showing the patterns seen of surface wear and removal on the extendable telescopic rod. a, b Show the pattern of surface wear on the same rod at different orientation, while $\mathbf{c}$ shows a different pattern of surface degradation on another rod

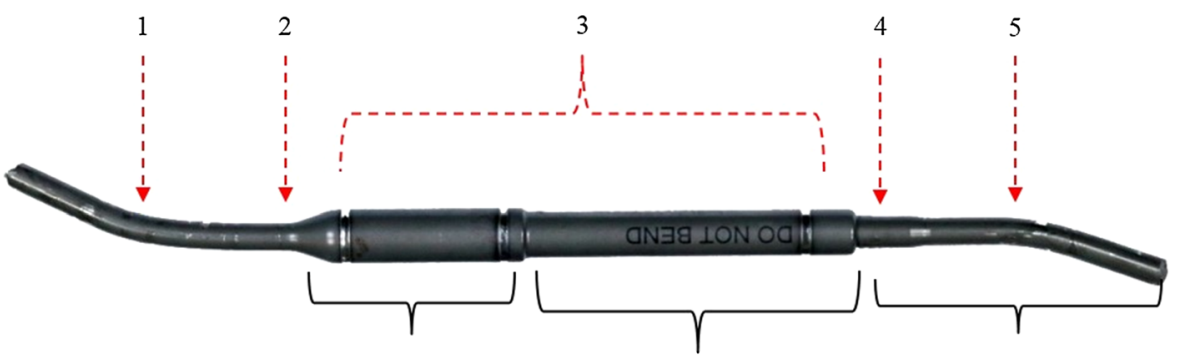

Actuator - Magnet Actuator-Sleeve Extendable Telescopic Rod

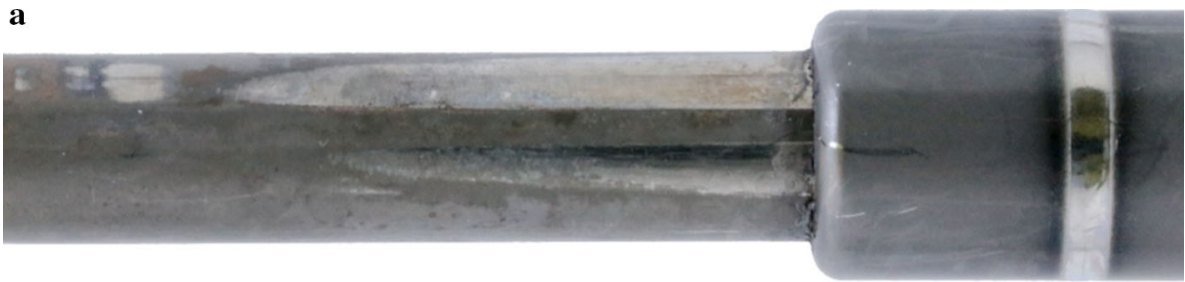

b

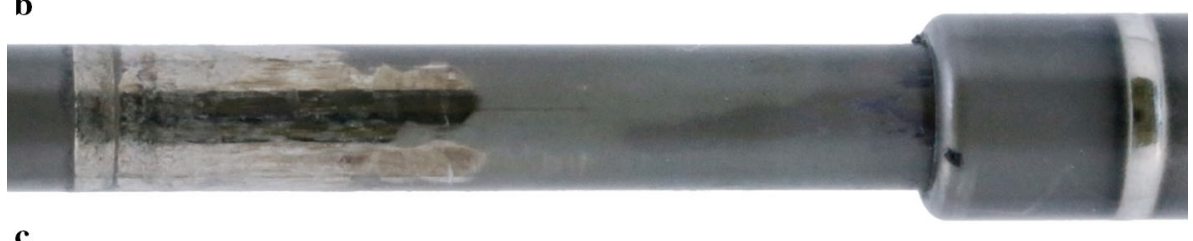

c

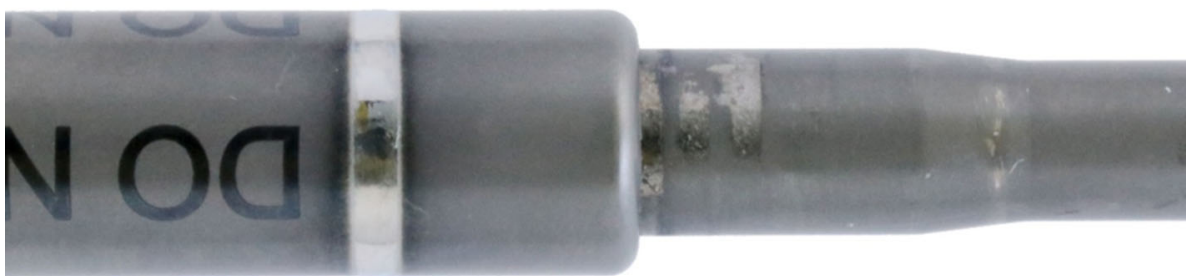




\section{Macroscopic inspection}

All retrieved implants showed signs of surface degradation on the extendable telescopic rod on regions that had been extended during the lengthening procedure and showed surface damage in two predominant patterns (see Fig. 4ac). All implants showed some evidence of scratches attributed to surgical tools used during implantation and revision surgery. The results of grading the areas of interest in terms of surface damage are shown in Table 3. Table 4 presents the findings from the macroscopic inspection (type of pattern at the junction) with the results from the radiographs (fractured or not mechanism). All rods with fractured pins had the same wear pattern at the junction, removing a larger area of the surface layer than the MCGRs with an intact internal mechanism.

\section{Microscopic inspection}

All implants showed signs of pitting and fretting on areas of the rod exposed during distraction, Fig. 5a-c.

Table 3 Breakdown of the scores (0-3) of surface damage for each area of interest for each rod

\begin{tabular}{llllll}
\hline Patient-rod & Area 1 & Area 2 & Area 3 & Area 4 & Area 5 \\
\hline 1-A & 2 & 2 & 0 & 2 & 2 \\
1-B & 1 & 0 & 0 & 2 & 2 \\
2-A & 2 & 1 & 1 & 2 & 3 \\
2-B & 1 & 0 & 1 & 2 & 1 \\
$3-A$ & 1 & 0 & 1 & 2 & 1 \\
$3-B$ & 1 & 1 & 1 & 2 & 1 \\
4 & 1 & 1 & 1 & 2 & 1 \\
$5-A$ & 2 & 1 & 1 & 2 & 0 \\
$5-B$ & 1 & 0 & 1 & 2 & 1 \\
\hline
\end{tabular}

\section{Forensic analysis of two selected rods}

\section{Micro-CT}

MCGR with fractured pin-mCT of the MCGR showed the drive shaft had become disengaged from the magnet, Fig. 6. Fragments of the pin were located around the ball bearing and had fractured into two pieces. Two of the fragments were located within the plain ball bearing with the remaining four on top, Fig. 6a. MCGR with intact pin$\mathrm{mCT}$ confirmed that the magnet and the drive shaft were still engaged, with the pin in place, Fig. 6c.

\section{Mechanical sectioning}

The MCGR with the fractured pin was sectioned in two areas: (1) one to allow access to the site of the fractured pin and (2) the other to access the thrust ball bearing and magnet within the sleeve, Fig. 7a, b. We found that the pin was fractured into six pieces, and once sectioned, the pieces were located in the same region, Fig. $6 a, b$.

Visual and microscopic inspection showed debris and deposits on the bearings (see Fig. 8a-d). The lengthening component of the rod was inseparable from the caudal part of the implant, unable to move due to large build up of debris, Fig. 9.

The intact rod was sectioned in two parts, to reveal the train drive mechanism, which is not visible at the failed rod, Fig. 10a. There were also signs of debris at the interior of the intact rod although not in the same quantity as the pin fractured rod, Fig. 10b, c.

\section{$S E M / E D X$}

SEM confirmed the presence of debris on the pin, on the train drive mechanism and on the exterior lengthening rod. The fractured surfaces of the actuator pin were rough with

Table 4 Type of surface damage per rod, classified during macroscopic inspection according to the patterns in Fig. 4, combined with information about fractured pin from imaging

\begin{tabular}{|c|c|c|c|c|c|}
\hline Patient-rod & $\begin{array}{l}\text { Damage pattern } \\
\text { at area } 4\end{array}$ & $\begin{array}{l}\text { Presence of a fractured } \\
\text { pin in the radiograph }\end{array}$ & $\mathrm{mCT}$ or X-ray & $\begin{array}{l}\text { Macroscopic and } \\
\text { microscopic inspection }\end{array}$ & $\begin{array}{l}\text { SEM and } \\
\text { EDX }\end{array}$ \\
\hline $1-\mathrm{A}$ & $a$ and $b$ & Yes & $\mathrm{mCT}$ & Yes & Yes \\
\hline $1-\mathrm{B}$ & $\mathrm{c}$ & No & $\mathrm{mCT}$ & Yes & Yes \\
\hline $2-\mathrm{A}$ & $\mathrm{c}$ & No & X-ray & Yes & No \\
\hline $2-\mathrm{B}$ & None & No & X-ray & Yes & No \\
\hline $3-\mathrm{A}$ & $\mathrm{c}$ & No & X-ray & Yes & No \\
\hline $3-\mathrm{B}$ & $\mathrm{c}$ & No & X-ray & Yes & No \\
\hline 4 & $\mathrm{~b}$ & Yes & X-ray & Yes & No \\
\hline $5-\mathrm{A}$ & $\mathrm{b}$ & Yes & X-ray & Yes & No \\
\hline $5-B$ & c & No & X-ray & Yes & No \\
\hline
\end{tabular}




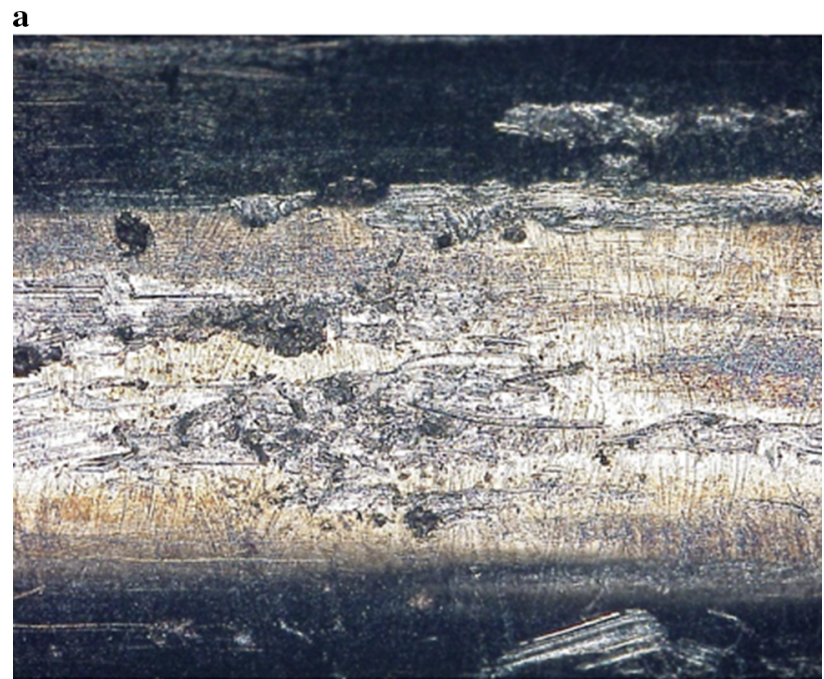

b

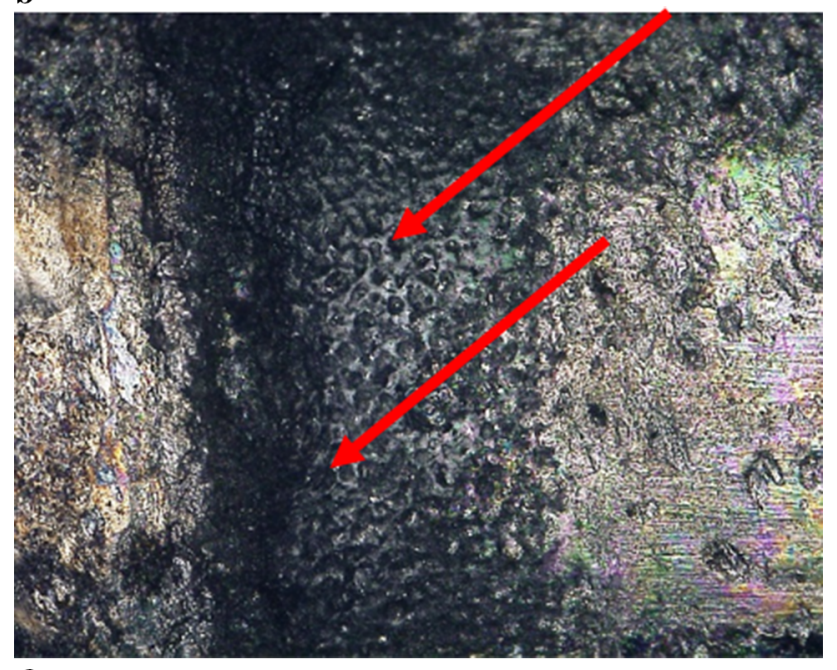

c

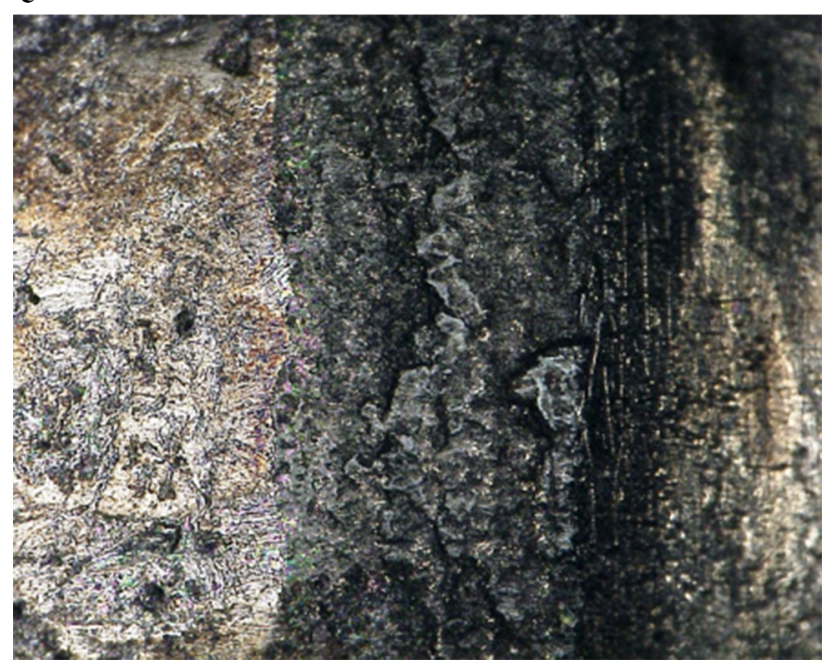

Fig. 5 a Microscopic picture of the damaged surface near the junction at $\times 200$ magnification. b Microscopic picture of the damaged surface near the junction at $\times 200$ magnification, with red arrows showing the pitting. c Microscopic picture of the damaged surface near the junction at $\times 200$ magnification
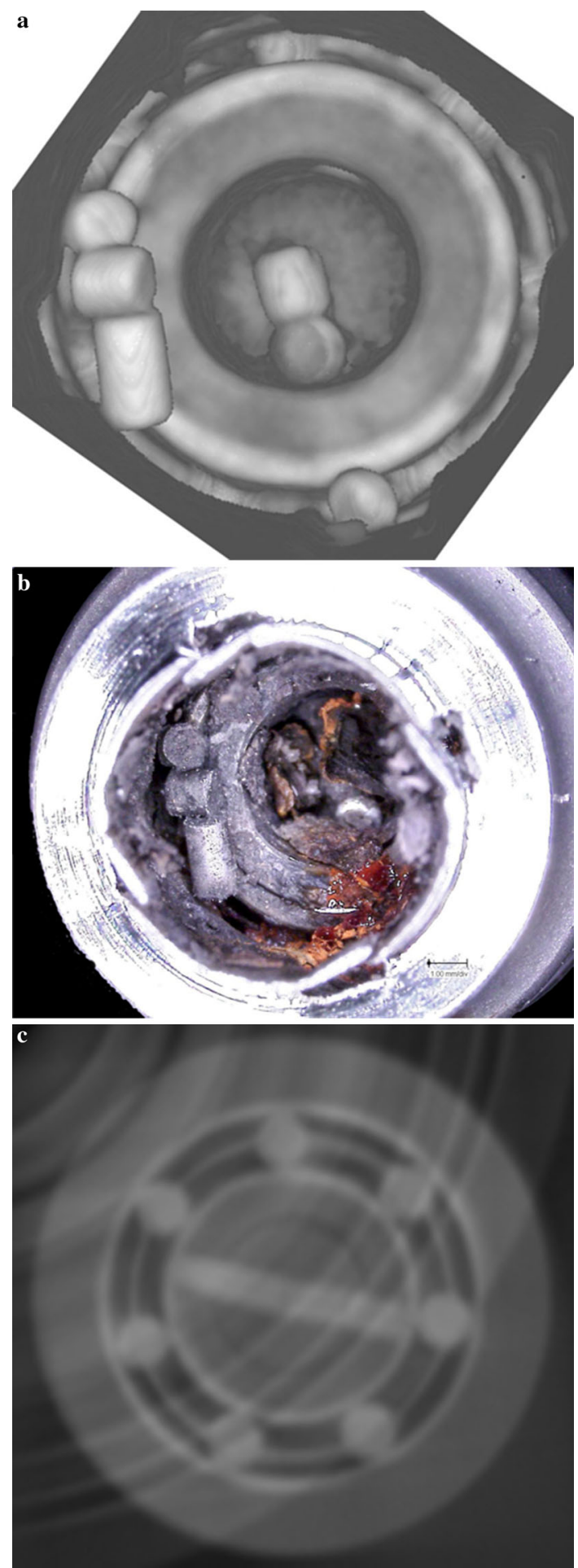

Fig. 6 a Micro-CT image of the fractured pin in and atop of the ball bearing. b Image of sectioned rod showing the fractured pin in and atop of the ball bearing $\mathbf{c}$ Micro-CT image of the intact pin 
a

(a)

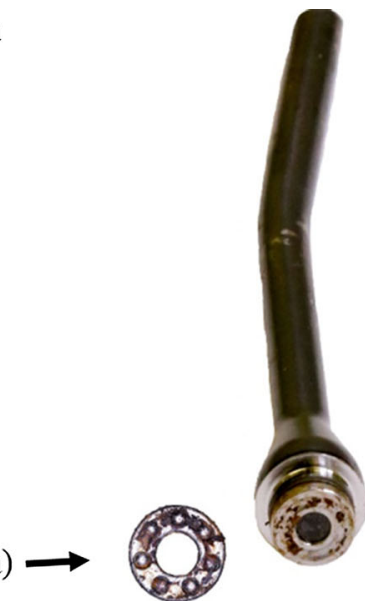

(b)
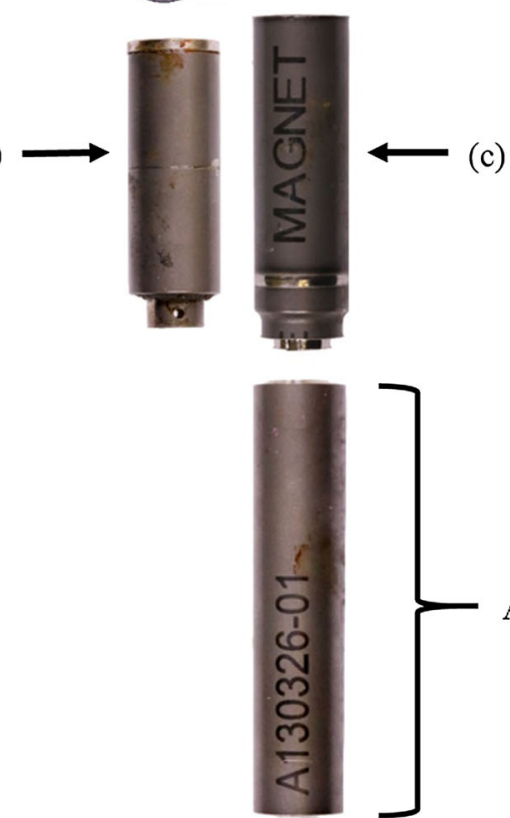

(d)

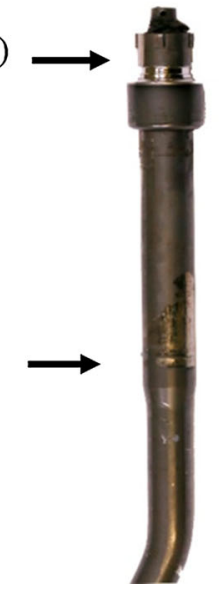

Fig. 7 a Image of all parts of the fractured rod after sectioning. (a) Thrust bearing $(b)$ magnet $(c)$ keeper plate $(d)$ ball bearing $(e)$ drive train screw $(f)$ fixed nut $(g)$ extendable telescopic rod. b

Actuator - Sleeve

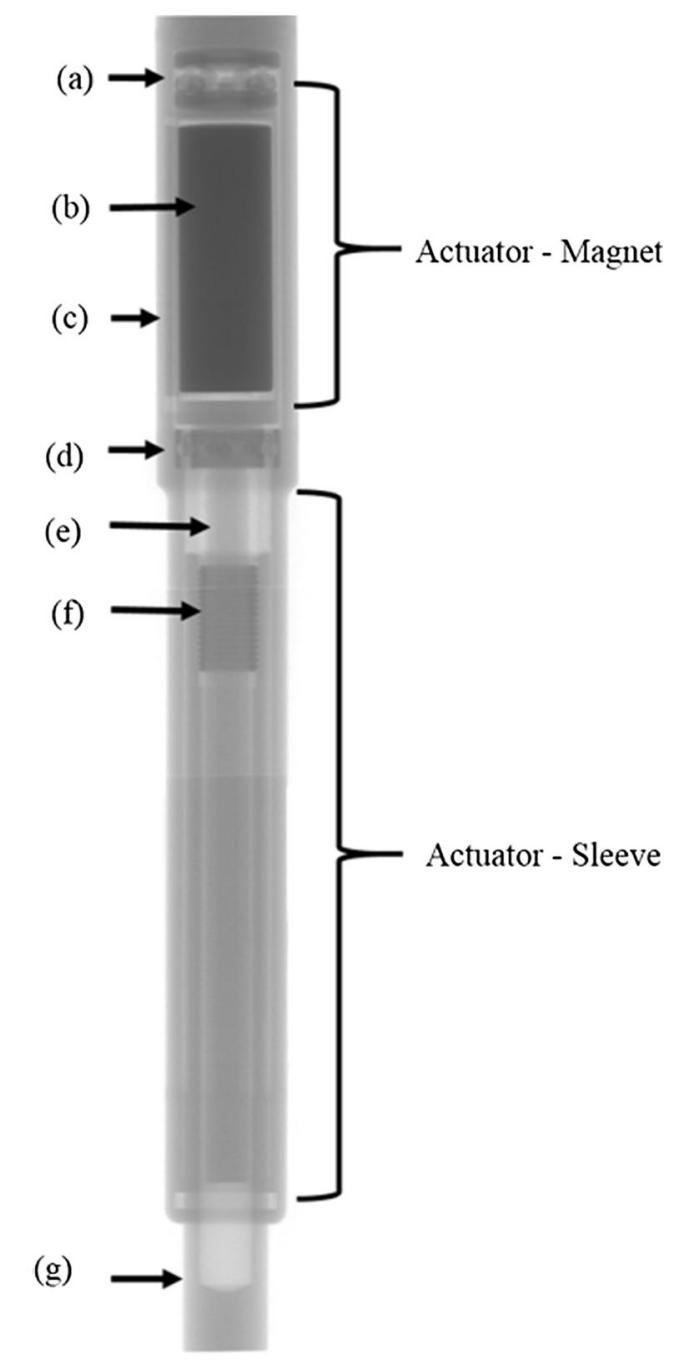

b Radiograph showing the construct of an intact spinal rod. (a) Thrust bearing $(b)$ magnet $(c)$ keeper plate $(d)$ ball bearing $(e)$ drive train screw $(f)$ fixed nut $(g)$ extendable telescopic rod 
Fig. 8 a Microscopic image of the distal end of the magnet where thrust bearing articulates showing corrosive debris $(\times 50$ magnification). b Microscopic image of the distal end of the magnet where thrust bearing articulates showing corrosive debris $(\times 100$ magnification). c Microscopic picture of the thrust bearing showing signs of corrosion ( $\times 50$ magnification). d Microscopic picture of the thrust bearing showing signs of corrosion $(\times 100$ magnification $)$
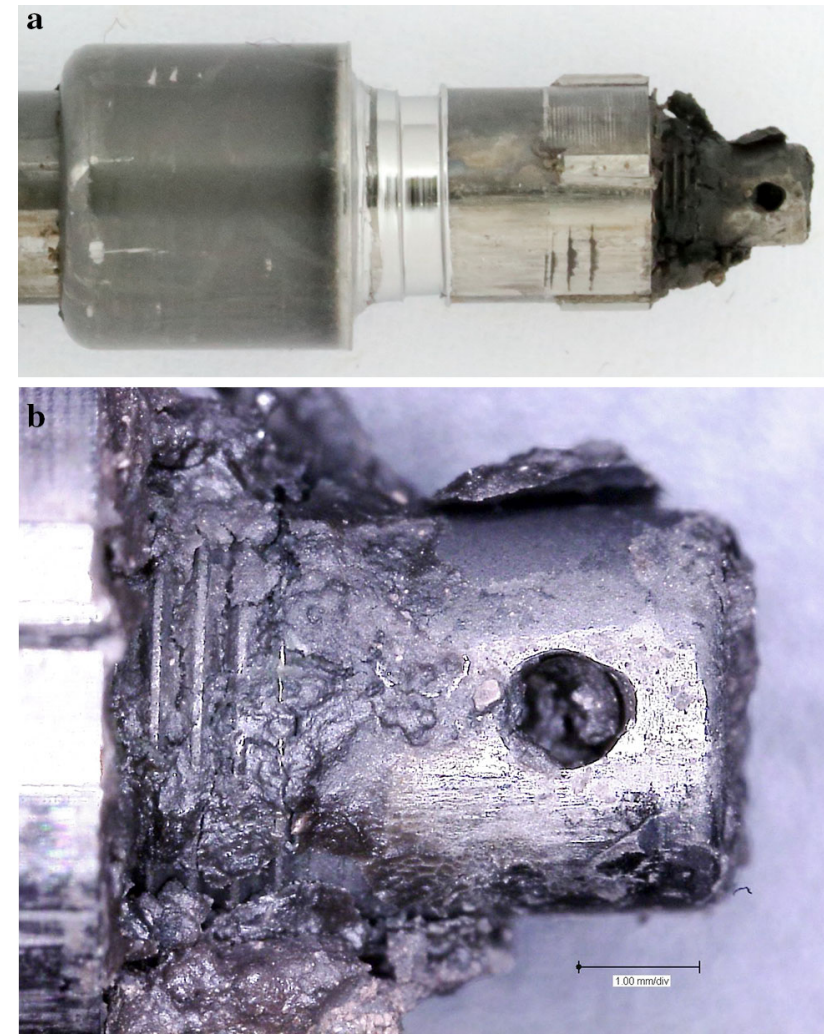

Fig. 9 a Image showing drive train pin hole and debris. b Microscopic image showing drive train pin hole and debris $(\times 30$ magnification)

no clear indication of the exact location of fracture initiation. The rough morphology of the fractured areas did not account for any fatigue-related cracking. EDX analysis suggested that the pin was made of ferritic stainless steel, but was highly corroded (Fig. 11-c); we found traces of sulphur $(0.2 \%)$, calcium $(0.12 \%)$ and titanium (Fig. 11c). Specifically, oxygen amount was more than double on the corroded regions of the actuator pin compared to the original areas (60 versus $30 \%)$. On the corroded region, we noticed a tenfold decrease of the amount of iron and a fivefold reduction of the chromium content compared to non-corroded areas. The presence of titanium (5\%), aluminium $(0.4 \%)$, and vanadium $(0.4 \%)$ suggested transfer of metal debris from mechanical wear of the rod to the actuator pin. We confirmed that the rod (Fig. 12a, b) and the drive mechanism (Fig. 13a-c) were made of titanium
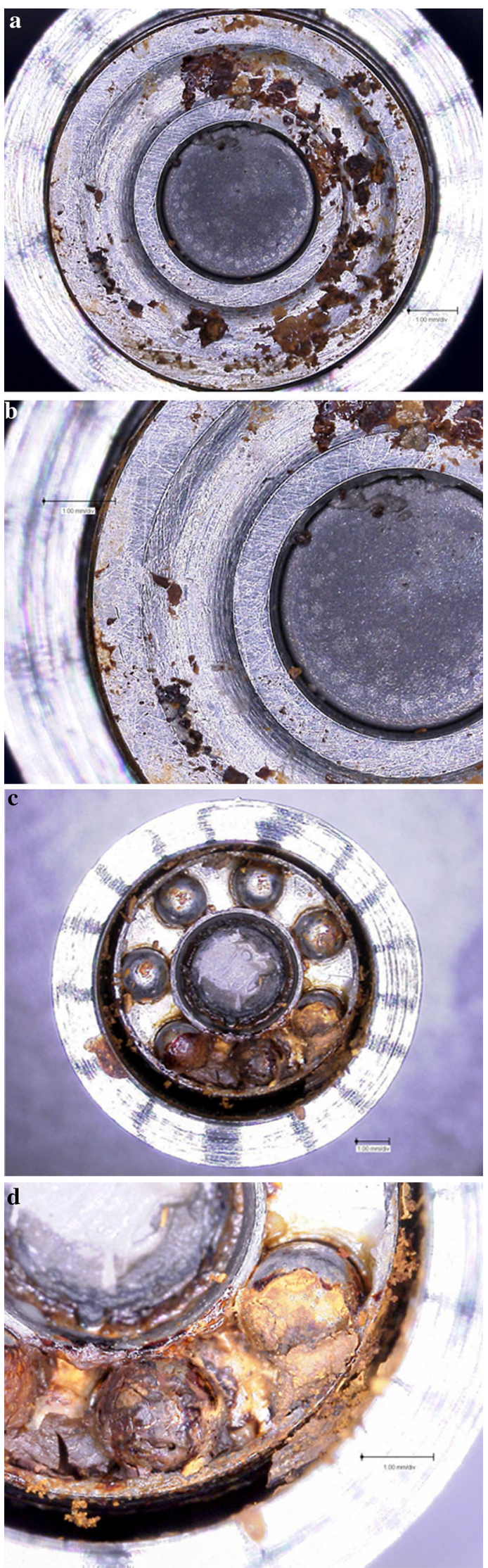
Fig. 10 a Train mechanism as revealed from sectioning the intact magnetic rod. b Train drive mechanism of the intact rod under the microscope $(\times 20$ magnification). c Microscopic picture of the junction of the intact rod showing signs of debris ( $\times 50$ magnification)

\section{a}

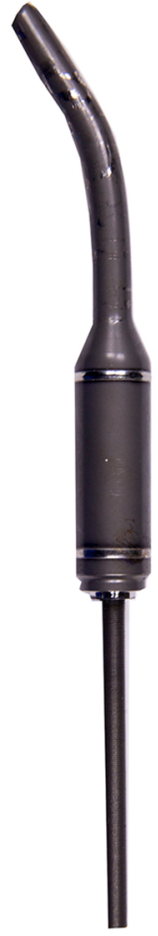

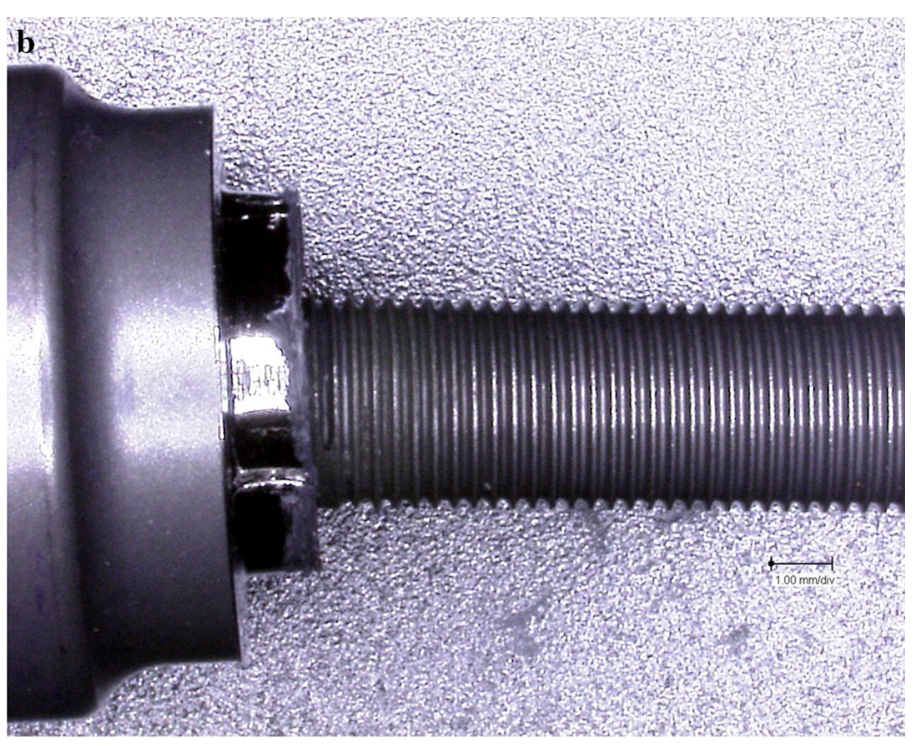

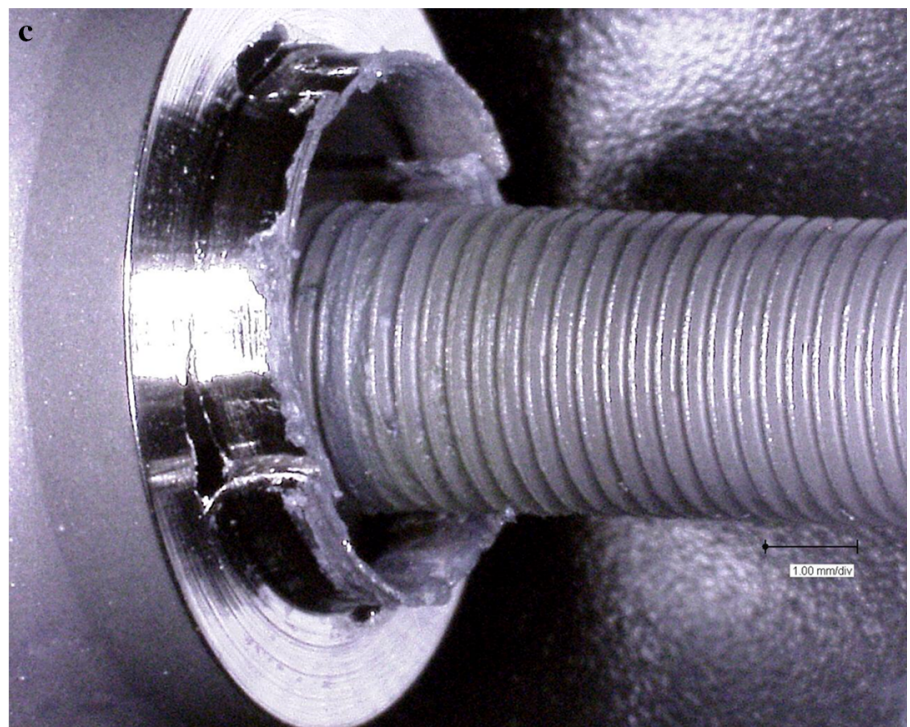

alloys and that both had evidence of corrosive products containing sulphur $(0.15$ and $0.6 \%$, respectively), iron ( $1 \%$ in the case of the rod), phosphorus (6 and $0.4 \%$, respectively), and calcium (Figs. 12b, 13c). EDX analysis of the debris on the drive mechanism revealed the presence of sodium $(0.6 \%)$, potassium $(0.2 \%)$, and chlorine $(0.4 \%)$, confirming the presence of body fluids inside the magnetic rod.

\section{Discussion}

The use of MCGRs for the treatment of EOS is increasing in popularity as it is non-invasive (post implantation) way and is a less traumatic experience for young patients compared to the traditional growing rods [10, 11, 20-22]. A recent clinical study suggests that patients previously treated with the traditional growing rods can be converted to MCGRs with comparable positive results [12]. Clinical cases reporting pin fracture and failure to distract have been published over the last 2 years [11-15]; this is the first retrieval paper using state-of-the-art forensic analysis to determine the mechanism leading to pin fracture.

We examined nine retrieved MCGRs and found that a third of them had a fractured pin. The surface damage seen on the extendable telescopic component of all implants we examined suggests that this surface is being scratched by the lip of the sleeve during the lengthening procedure. The pattern of the damaged surface on the MCGRs with intact pins is different to the pattern of the damaged surface of the 

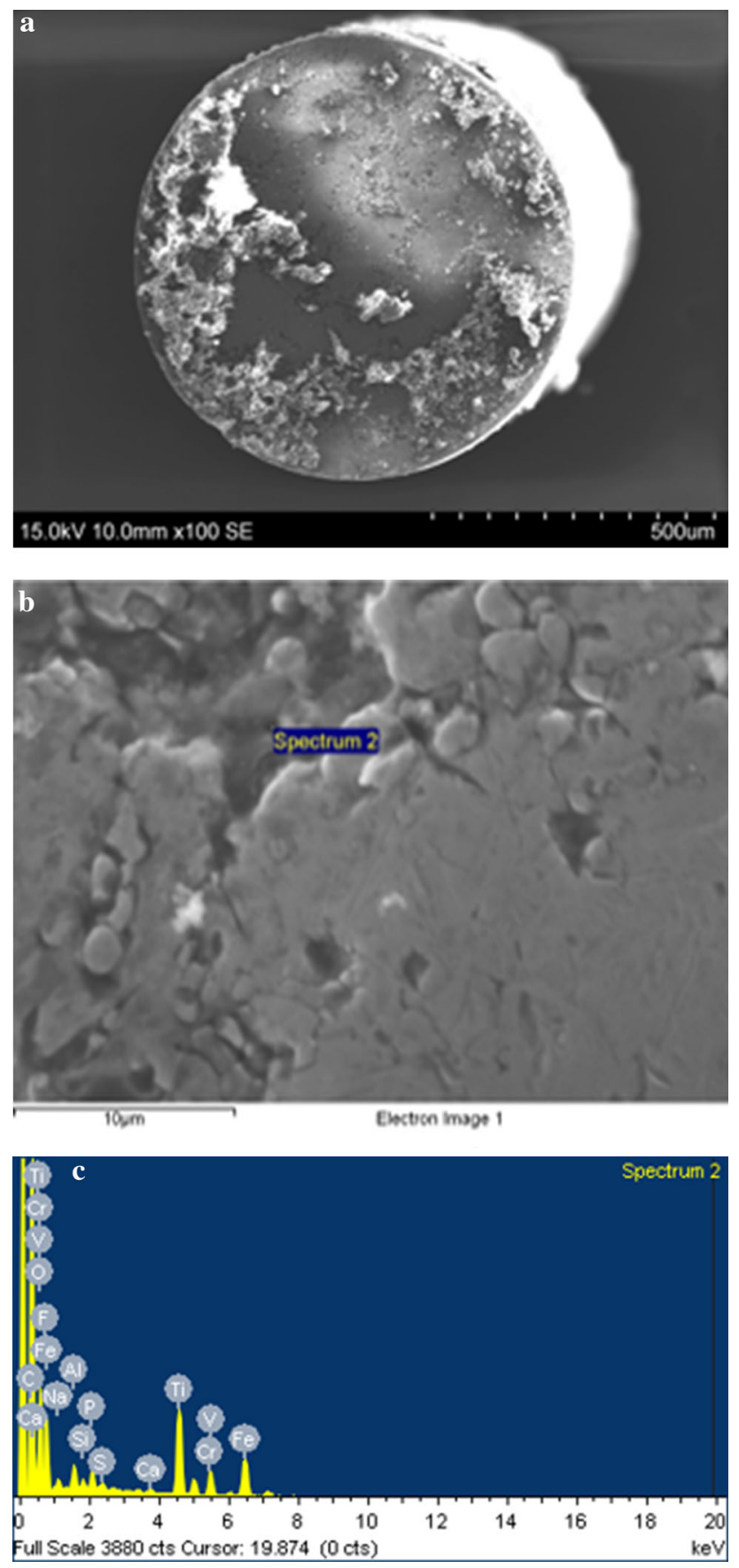

Fig. 11 a SEM image on the internal pin. b SEM image on the internal pin. $\mathbf{c}$ EDX analysis of debris on the internal pin

MCGRs with fractured pins. The regularity of the distribution of scratches in MCGRs with intact pins (Fig. 4c) likely reflects the number of distraction therapies. In contrast, the irregular patterns of surface damage (Fig. 4a, b) were only seen in cases with fracture of the pin. The surface damage seems to be linked to the bending moment.
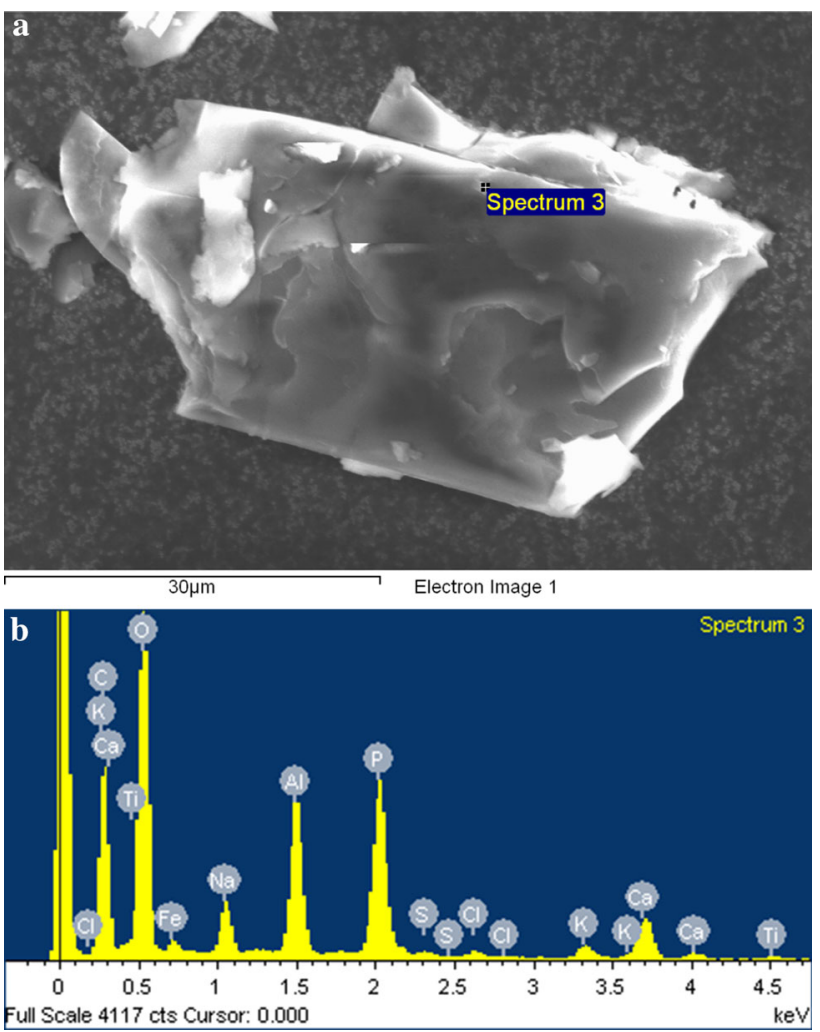

Fig. 12 a SEM image of debris on the external damaged surface of the rod on a carbon coated tab. $\mathbf{b}$ EDX analysis of the debris on the external damaged surface of the rod

We speculate that the gap between the telescopic component of the rod and external shell allowed for fluid ingress into the interior of the actuator in all rods. Evidence of both biological and corrosive debris on the thread mechanism supports the hypothesis of fluid ingress. Analysis of this debris (Fig. 13a) showed a high amount of $\mathrm{TiO}_{2}$ which the surface of the MCGR is comprised of. This included traces of sulphur, iron, phosphorus, and calcium, suggesting a corrosive origin of the debris. The fluid ingress and the debris build up inside the actuator is likely to have resulted in increased the friction between the moving components of the actuator, while, at the same time, reducing its capability to distract. We suggest that the combination of these two mechanisms could lead to the fracture of the actuator pin. Reports on different failure modes of spinal devices have been published [23], which are caused by fatigue during clinical use, in contrast to the findings in this paper which are mainly implant related.

The most current update from National Institute of Health and Care Excellence (NICE) states that there have been only two alterations to the original design of the MCGR [9]; in 2010, the addition of a keeper plate to prevent the internal magnet from rotating, and in 2012, the 

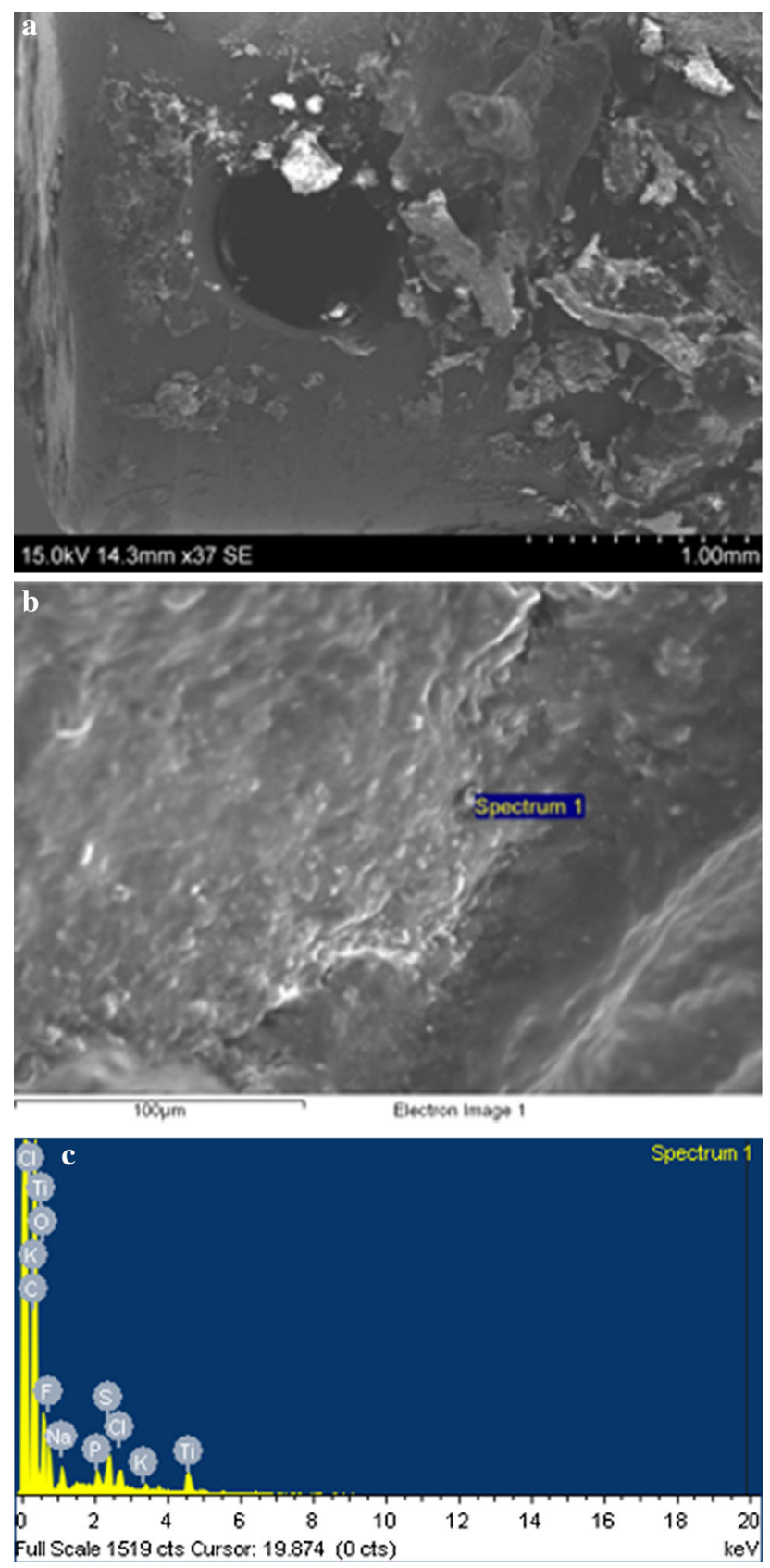

Fig. 13 a SEM picture of debris on the drive mechanisms of the fractured rod. b SEM picture of debris on the drive mechanisms of the fractured rod. c EDX analysis of debris (graph on the right) on the drive mechanism of the fracture rod

change of the welding process to prevent implant fracture. A recent 4 year follow-up mentioned the company's intention to increase the pin's diameter [15]. However, we could not find any design updates regarding the shielding of the internal mechanism from fluid ingress. Future study of periprosthetic tissue will determine whether corroded material migrated from the internal mechanism to the outside.
This paper offers further insights into the mechanism of pin fracture and distraction failure, but does not compensate for all modes of failure of the rods. It is important to understand more about the potential patient (underlying diagnoses and curvatures) and surgical (distraction techniques and protocols) risk factors of these cases with pin fractures to give more insight as to why and how this has occurred.

\section{Conclusion}

This is the first retrieval study to investigate the mechanisms of failure in MCGRs. We examined a series of nine retrieved rods and found that a third had failed due to pin fracture secondary to corrosion of the internal mechanism.

We recommend that surgeons consider that any inability of MCGRs to distract may be due to debris building up inside the mechanism, thereby preventing normal function.

Acknowledgements We are grateful for the support of the theatre staff at the Royal National Orthopaedic Hospital, Great Ormond Street and The Wellington Hospital for their help in collecting and delivering the implants to us, Mr. A. Yasen for providing us with spine implants and relevant clinical information and for consenting patients, Gwynneth Lloyd, Elizabeth Ellis, Anna Di Laura and Akramul Hoque for their coordination of the retrieval centre, Dr. Paul Bills, from the University of Huddersfield, for the micro-CT of two of the spine implants, Dr. Tom Gregory, SEM technician, at UCL Archaeology Institute and Keith Rayner for technical support, Tony Turner and Ymma Pennicard from the Radiology Department of the Royal National Orthopaedic Hospital. One author received funding from K2M for collection and analysis of failed implants. Two authors received funding from the British Orthopaedic Association through an industry consortium of nine manufacturers: DePuy International Ltd (Leeds, UK), Zimmer GmbH (Winterthur, Switzerland), Smith and Nephew UK Ltd (Warwick, UK), Biomet UK Ltd (Bridgend, South Wales, UK), JRI Ltd (London, UK), Finsbury Orthopaedics Ltd (Leatherhead, UK), Corin Group PLC (Cirencester, UK), Mathys Orthopaedics Ltd (Alton, UK), and Stryker UK Ltd (Newbury, UK).

\section{Compliance with ethical standards}

Conflict of interest There is no conflict of interests.

Open Access This article is distributed under the terms of the Creative Commons Attribution 4.0 International License (http://crea tivecommons.org/licenses/by/4.0/), which permits unrestricted use, distribution, and reproduction in any medium, provided you give appropriate credit to the original author(s) and the source, provide a link to the Creative Commons license, and indicate if changes were made.

\section{References}

1. Gillingham BL, Fan RA, Akbarnia BA (2006) Early onset idiopathic scoliosis. J Am Acad Orthop Surg 14(2):101-112

2. Campbell RM, Smith MD, Mayes TC, Mangos JA, WilleyCourand DB, Kose N et al (2003) The characteristics of thoracic 
insufficiency syndrome associated with fused ribs and congenital scoliosis. J Bone Jt Surg Am 85(3):399-408

3. Goldberg C, Gillic I, Connaughton O, Moore D, Fogarty E, Canny $G$ et al (2003) Respiratory function and cosmesis at maturity in infantile-onset scoliosis. Spine 28(20):2397-2406

4. Cunin V (2015) Early-onset scoliosis-current treatment. Orthop Traumatol Surg Res 101(1):S109-S118

5. Mehta M (2005) Growth as a corrective force in the early treatment of progressive infantile scoliosis. J Bone Jt Surg $\mathrm{Br}$ 87(9):1237-1247

6. Yoon WW, Sedra F, Shah S, Wallis C, Muntoni F, Noordeen H (2014) Improvement of pulmonary function in children with early-onset scoliosis using magnetic growth rods. Spine. 39(15):1196-1202

7. La Rosa G, Oggiano L, Ruzzini L (2015) Magnetically controlled growing rods for the management of early-onset scoliosis: a preliminary report. J Pediatr Orthop. doi:10.1097/BPO.00000000 00000597

8. Hickey B, Towriss C, Baxter G, Yasso S, James S, Jones A et al (2014) Early experience of MAGEC magnetic growing rods in the treatment of early onset scoliosis. Eur Spine J 23(1):61-65

9. Jenks M, Craig J, Higgins J, Willits I, Barata T, Wood $\mathrm{H}$ et al (2014) The MAGEC system for spinal lengthening in children with scoliosis: a NICE Medical Technology Guidance. Appl Health Econ Health Policy 12(6):587-599

10. Akbarnia BA, Marks DS, Boachie-Adjei O, Thompson AG, Asher MA (2005) Dual growing rod technique for the treatment of progressive early-onset scoliosis: a multicenter study. Spine 30(17S):S46-S57

11. Cheung KMC, Cheung JPY, Samartzis D, Mak KC, Wong YW, Cheung WY et al (2012) Magnetically controlled growing rods for severe spinal curvature in young children: a prospective case series. Lancet 379(9830):1967-1974

12. Keskinen H, Helenius I, Nnadi C, Cheung K, Ferguson J, Mundis G, Pawelek J, Akbarnia BA (2016) Preliminary comparison of primary and conversion surgery with magnetically controlled growing rods in children with early onset scoliosis. Eur Spine $\mathbf{J}$ 25(10):3294-3300
13. Stokes OM, O’ Donovan EJ, Samartzis D, Bow CH, Luk KDK, Cheung KMC (2014) Reducing radiation exposure in early-onset scoliosis surgery patients: novel use of ultrasonography to measure lengthening in magnetically-controlled growing rods. Spine J 14(10):2397-2404

14. Yoon WY, Chang AC, Tyler P, Butt S, Raniga S, Noordeen H (2015) The use of ultrasound in comparison to radiography in magnetically controlled growth rod lengthening measurement: a prospective study. Eur Spine J 24:1422-1426

15. Teoh KH, Winson DM, James SH, Jones A, Howes J, Davies PR et al (2016) Magnetic controlled growing rods for early onset scoliosis: a 4-year follow up. Spine J 16(4 Suppl):S34-S39

16. Jones CS, Stokes OM, Patel SB, Clarke AJ, Hutton M (2015) Actuator pin fracture in magnetically controlled growing rods: two cases. Spine J 16(4):e287-e291

17. Cheung JP, Cahill P, Yaszay B, Akbarnia BA, Cheung KMC (2015) Special article: update on the magnetically controlled growing rod: tips and pitfalls. J Orthop Surg 23(3):383-390

18. Annual Meeting of the British Scoliosis Society (2016)

19. Akazawa T, Minami S, Takahashi K, Kotani T, Hanawa T, Moriya H (2005) Corrosion of spinal implants retrieved from patients with scoliosis. J Orthop Sci 10(2):200-205

20. Bess S, Akbarnia BA, Thompson GH, Sponseller PD, Shah SA, El Sebaie $\mathrm{H}$ et al (2010) Complications of growing-rod treatment for early-onset scoliosis. J Bone Jt Surg Am 92(15):2533-2543

21. Caldas JCS, Pais-Ribeiro JL, Carneiro SR (2004) General anesthesia, surgery and hospitalization in children and their effects upon cognitive, academic, emotional and sociobehavioral development-a review. Pediatr Anesth 14(11):910-915

22. Yang JS, Sponseller PD, Thompson GH, Akbarnia BA, Emans JB, Yazici M et al (2011) Growing rod fractures: risk factors and opportunities for prevention. Spine. 36(20):1639-1644

23. Yamanaka K, Mori M, Yamazaki K, Kumagai R, Doita M, Chiba A (2015) Analysis of the fracture mechanism of Ti-6Al-4V alloy rods that failed clinically after spinal instrumentation surgery. Spine 40(13):E767 